Relations industrielles

Industrial Relations

\title{
Les dynamiques de la PME : approches internationales sous la direction de Bruno Courault et Philippe Trouvé, Paris : \\ Presses universitaires de France, 2000, 404 p., ISBN \\ 2-11-088612-9.
}

\section{Jean Saglio}

Volume 57, numéro 2, printemps 2002

URI : https://id.erudit.org/iderudit/006796ar

DOI : https://doi.org/10.7202/006796ar

Aller au sommaire du numéro

Éditeur(s)

Département des relations industrielles de l’Université Laval

ISSN

0034-379X (imprimé)

1703-8138 (numérique)

Découvrir la revue

Citer ce compte rendu

Saglio, J. (2002). Compte rendu de [Les dynamiques de la PME : approches internationales sous la direction de Bruno COURAULT et Philippe Trouvé, Paris : Presses universitaires de France, 2000, 404 p., ISBN 2-11-088612-9.]

Relations industrielles / Industrial Relations, 57(2), 415-416.

https://doi.org/10.7202/006796ar

Tous droits réservés @ Département des relations industrielles de l’Université Laval, 2002
Ce document est protégé par la loi sur le droit d'auteur. L'utilisation des services d'Érudit (y compris la reproduction) est assujettie à sa politique d'utilisation que vous pouvez consulter en ligne.

https://apropos.erudit.org/fr/usagers/politique-dutilisation/ 
critical reader yearns for more, well, evidence, of this assertion. Likewise, though the comments of the nurse interviewees are cogent, one wishes that the authors had done even limited verification to solidify the analysis. For example, the authors report "nurse after nurse" stating that infection rates in hospitals are rising because of cutbacks in housekeeping. While most readers would be ready to believe this assertion, both the statistics and the causation could easily have been corroborated.

Parenthetically, it would be remiss not to reproach both the authors and the publisher for the absence of an index, a complaint that is not exclusive to this book and this publisher. If one is producing serious social science publications, to which readers might wish to make further reference, the absence of an index is inexcusable. Notwithstanding these complaints, there is more than enough in this book to worry a reader about the consequences of health care reform on workers, patients and their families, even before the change of government.

LARRY HaIVEN Saint Mary's University

\section{Les dynamiques de la PME : approches internationales}

sous la direction de Bruno Courault et Philippe Trouvé, Paris : Presses universitaires de France, 2000, 404 p., ISBN 2-11-088612-9.

La prise en compte dans les analyses économiques et sociologiques du rôle des entreprises de faible taille dans le développement économique est un phénomène relativement récent en France. Même si l'ampleur de ce mouvement reste marginale, un certain nombre de programmes de recherches ont été mené sur ces thèmes depuis une quinzaine d'années. Cet ouvrage collectif est le produit ultime d'un séminaire de recherches de novembre 1997 organisé par la DARES au ministère de l'Emploi et de la Solidarité pour faire le point de quelques-uns de ces travaux, à dominante sociologique.

Une première partie de l'ouvrage réunit des analyses faites sous des angles variés. Les terrains d'enquête, les thématiques de recherche, aussi bien que les paradigmes explicatifs ne sont ni complémentaires ni vraiment articulés. La juxtaposition de ces analyses variées n'est cependant ni fortuite ni dénuée d'intérêt. Elle enseigne que, si l'analyse des PME ne peut constituer en soi un domaine particulier de la sociologie économique, la prise en compte de ce qui s'y passe permet le plus souvent d'enrichir les débats et de renouveler les analyses. La question de l'inscription territoriale, sans être véritablement traitée de front, y est abordée sous des angles variés et cette approche permet d'infirmer une fois encore la relation fréquemment avancée entre inscription locale et marché limité.

L'ensemble des contributions présentées dans la seconde partie porte, à l'exception de la dernière, sur le cas des industries de l'habillement. La comparaison internationale, réalisée par la proximité des contributions, est ici particulièrement féconde pour montrer comment se mettent en place les stratégies de restructuration et d'adaptation aux nouvelles donnes des marchés. Dans la plupart de ces cas, on voit combien l'analyse fine et située, par des observations faites sur longue période, permet de dépasser les limites des outils d'analyse classique en reconstruisant, cas par cas, les éléments d'analyse pertinents.

Ces deux ensembles de contributions originales sont encadrés par deux textes de synthèse signés par les coordonnateurs. Le premier est une présentation de la littérature existante sur ces sujets principalement en France et secondairement en Europe. Le second porte sur la 
complexité, voire l'indécidabilité du jugement sur la contribution des PME à l'emploi.

Longtemps, l'attention portée par les chercheurs aux entreprises de faible taille a été négligeable. Seules les grandes entreprises méritaient intérêt et attention et c'était en observant les stratégies et les comportements dans ces organisations que l'on pouvait tirer des enseignements valables pour l'avenir. La perspective est aujourd'hui renversée, au moins pour une part. Comment expliquer ce changement et son importance ? Les coordonnateurs de la recherche abordent cette question dans l'introduction : l'explication par la conjoncture économique et la crise du modèle taylorien-fordien - qu'ils présentent comme explication principale est incontestablement recevable. Suffitelle pour autant? Il serait de mauvaise foi de prêter aux auteurs une réponse catégorique. On est cependant surpris de constater l'absence de contributions d'historiens ou de travaux historiques fortement documentés. On peut encore remarquer qu'en privilégiant l'explication par les conjonctures économiques, ils exonèrent la collectivité des analystes - plus notamment français — de son retard indéniable à accepter la prise en compte du phénomène. La contribution des PME à l'emploi, remarque Philippe Trouvé dans son excellente conclusion, est une relation particulièrement complexe : en rendre compte exige à tout le moins d'avoir une vision dynamique et non statique des situations économiques. Une telle remarque n'estelle pas, par elle-même, une critique des modèles implicites sur lesquels sont construits les systèmes d'information économique ?

On peut encore regretter, notamment pour une publication patronnée par le ministère du travail, que la question de la spécificité des régulations de relations professionnelles dans ces entreprises ou ensembles d'entreprises n'ait pas été véritablement traitée pour elle-même. On sait que les acteurs syndicaux éprouvent aujourd'hui encore les plus grandes difficultés à subsister dans ces entreprises. La présence syndicale y est nettement moins dense que dans les grandes entreprises et les administrations. Ce phénomène n'est pour autant pas explicable par le seul facteur de la taille des entreprises : au début du $\mathrm{XX}^{\mathrm{e}}$ siècle, le syndicalisme ouvrier était bien souvent un syndicalisme de salariés de petites et moyennes entreprises, souvent urbaines. La régulation professionnelle des marchés du travail était, très probablement le modèle implicite auquel ces syndicalistes se référaient. L'abandon de la référence à ce modèle et l'utopie de l'unification du système français de relations professionnelles expliquent-t-ils la spécificité des relations professionnelles dans les PME si elle existe ? L'absence de ce thème ne peut manquer d'intriguer. En 1996, notent les auteurs (p. XLVIII), 77,6 \% des salariés appartiennent à des établissements de moins de 200 salariés. Et encore ne s'agit-il que du champ couvert par le régime d'assurance-chômage (l'Unédic). Si l'on prend encore en compte le fait que le quart des salariés français relèvent du secteur public, on déduira aisément que c'est moins d'un salarié sur cinq qui relève de ces grandes organisations privées dont le fonctionnement est érigé en norme pour la compréhension de la société salariale française et des évolutions des relations professionnelles. Travailler sur les PME sous l'égide du ministère qui, non content d'assurer la tutelle des relations professionnelles en France, en est encore un des acteurs majeurs aurait pu être l'occasion de s'interroger sur une telle focalisation intellectuelle et institutionnelle.

Jean Saglio

CRISTO/DGES, Grenoble 